\title{
Microbiological Profile of Severe Lower Respiratory Tract Infection in Intensive Care Unit of a Tertiary Care Center of Dhaka, Bangladesh
}

M. Abdul Mannan', M. Abul Kashem², Fazle Rabbi Mohammed ${ }^{3}$, Raihan Rabbani ${ }^{4}$, M. Motiul Islam ${ }^{5}$

\begin{abstract}
:
Background: Microbial resistance to antibiotics is nowadays a great threat to intensive care unit patients especially in severe pneumonia leading to high morbidity and mortality. Bacterial profile and antibiotic sensitivity pattern would help clinician in selecting more proper empirical therapy before isolation of microbes.

Materials and Methods: This retrospective study was performed among 210 patients of lower respiratory tract infection in intensive care unit in Square Hospitals Ltd, a tertiary care hospital in Dhaka. Sputum, tracheal aspirates or broncho alveolar lavage were cultured, identified and antibiotic sensitivity pattern performed by standard methods.

Results: Of 210 specimens, 150 (71\%) were culture positive and 60 (29\%) showed no growth. From 150 culture positives, 216 isolates were recovered, of which 7 specimen shows more than 2 organisms each, 52 specimen shows 2 isolates per specimen remaining 91 specimen with single isolates were recovered. The most common organisms in order of frequency were, Acinetobacter baumannii (24.0\%), Staphylococcus aureus (20.6\%), Klebsiella pneumoniae (19.3\%), Pseudomonas aeruginisa (19.3\%), Escherichia coli (14\%) and Candida albicans (13\%). A very high rate (83-93\%) of resistance was observed among Acinetobactor baumanni to Beta lactum and clavulanate, Cephalosporin, Quinolones and Carbapenem. No resistant was observed with Colistin. Linezolid and Vancomycin are most effective against methicillin resistant Staphylococcus aureus, Coagulase negative Staphylococcus aureus and Escherichia coli, where no resistance was found.

Conclusion: Farmenter and nonfermenters are the both common etiological agents of lower respiratory tract infections in this intensive care unit. There is high rate of resistance to commonly used cephalosporin and $\beta$-lactam- $\beta$-lactamase inhibitors and quinolone group of drugs. Acinetobacter baumannii, Pseudomonas aeruginisa and Klebsiella showed most sensitivity to Colistin and actually no resistance was found.
\end{abstract}

Keywords: Antimicrobial resistance, lower respiratory tract infection, intensive care unit.

\section{Introduction:}

Antimicrobial resistance in intensive care unit (ICU) is a global problem even in countries where extensive infection control measures are implemented. Lower respiratory tract (LRT) infection with resistant bacteria in patients of intensive

1. Dr. M. Abdul Mannan, MBBS, DA, MSc

Specialist, Intensive Care Unit

Square Hospitals Limited, Dhaka, Bangladesh.

2. Dr. M. Abul Kashem, FCPS, MS (Urology)

Assistant Professor

Sir Salimullah Medical College \& Mitford Hospital, Dhaka, Bangladesh

3. Dr. Fazle Rabbi Mohammed, MBBS, MD (Chest) Specialist, Department of Respiratory Medicine Square Hospitals Ltd, 18/F, BU Qazi Nuruzzaman Sarak, Dhaka, Bangladesh

4. Dr. Raihan Rabbani, FCPS (Medicine), MD(USA), Certified - American Board of Internal Medicine.

Consultant, ICU \& Internal Medicine

Square Hospitals Ltd. 18/F, BU Qazi Nuruzzaman Sarak, Dhaka 1205, Bangladesh.

5. Dr. M. Motiul Islam, MBBS

Clinical Staff, Intensive Care Unit

Square Hospitals Ltd. 18/F, BU Qazi Nuruzzaman Sarak, Dhaka 1205, Bangladesh. care units (ICUs) occurring in $20-25 \%$ of all ICU patients and resulting in high mortality (22-71\%) and morbidity. ${ }^{1-4}$ Inappropriate use of antibiotics in hospitals, community along with severity of illness, length of ICU stay, exposure to invasive devices, increased contact with health care personnel may contribute to the emergence of resistance..$^{5}$ It is gained by spontaneous mutation and acquiring new resistant gene from other species by horizontal transfer., ${ }^{3,4}$ esistance pattern vary from region to region depending on antibiotic use. ${ }^{6}$ An antibiotic policy avoids unnecessary use of broad spectrum antibiotics thereby decrease the development of resistant strains. $^{7}$

It is urgent to start proper empirical antibiotic before obtaining the microbial results, but emergence of multiple expanded spectrum of beta lactamase (ESBL) producing microbes and Multidrug resistant (MDR) pathogens, MRSA other categories of gram negative bacilli (GNB), make it difficult. Infectious disease society of America addressed three categories of gram negative bacilli, namely ESBL producing E. Coli and Klebsiella, MDR Pseudomonas and carbapenem resistant acinetobactor spp. are priority pathogen causing severity in ICU. ${ }^{8}$ Data on prevalent strains along with the susceptibility pattern can help in revising antibiotic policy and guiding clinicians for more proper antibiotic selection. ${ }^{9}$ Therefore, the present study was designed to know the bacterial profile and determine the antimicrobial resistance pattern among the microbes isolated from LRT of patients admitted to the ICU of Square Hospitals Ltd a 300 bedded tertiary care hospital in Dhaka city. 


\section{Materials and Methods:}

The present retrospective study was conducted in the microbiology department of Square Hospitals Ltd. in Dhaka during 2010-2011. Sputum, tracheal aspirates and broncho alveolar lavage (BAL) collected aseptically from 210 patients of most of them required mechanical ventilation. All samples were plated right after the collection and were further processed as per standard protocol. ${ }^{10}$ Single or mixed growth isolated from all the eligible consecutive samples were identified by observing the colony characteristics on the blood agar, Mac-Conkey agar plate and biochemical reactions using standard microbiological methods. ${ }^{10}$ Susceptibility testing was done by disc diffusion method. The following antibiotics (Hi-Media Disc in mcg) were tested: Amikacin, ciprofloxacin, moxifloxacin, aztreonem, colistin, tetracycline, cotrimoxazole, ceftazidime , ceftizoxime, meropenem, amoxycillin / clavulanic acid, imipenem piperacillin / tazobactum. Zone diameter was measured and interpreted as per the Clinical and Laboratory Standards Institute (CLSI) guidelines.

\section{Results:}

Out of 210 specimens, 150 (71\%) were culture positive whereas, $60(29 \%)$ specimens showed no growth. From the
150 culture positive specimens, 216 isolates were recovered. Out of 216 isolates, $60 \%$ were with single isolate, $35 \%$ were with two isolates $5 \%$ were with more than two isolates.

Table 1 represents the distribution of micro organisms recovered from the LRT specimens of ICU patients. The most common organism in order of frequency were Acinetobacter baumannii (23.6\%) Staph. aereous (20.7\%) P. aeruginosa $(19.6 \%)$, and Klebsiella pneumoniae (19.2\%) E. coli (14\%),Candida albicans (13\%), Coagulase negative staph aureus $(11.5 \%)$, Burkholderia cepacia (10\%). Entero coccus $(6 \%)$. Very high rate of resistance (83-93\%) was observed among $A$. baumannii with most of the antibiotics of beta lactum-clavulunate Penicillins, Cephalosporin, Quinolones, Aminoglycoside drugs. P. aeruginosa $(30-66 \%)$ and $K$. pneumoniae were (13-65\%) resistant to most of the commonly used antibiotics. Colistin was the most effective drug against $A$. baumannii, $K$. pneumoniae, and. $P$. aeruginosa. Linezolid and Vancomycin were the most effective drug against Staph. aerous, E. coli and Coagulase negative Staph. aereous and moderately sensitive (45-74\%) with Gentamycin, cotrimoxazole and tetracycline. 13-16\% Klebsiella pneumoniae are resistant to carbapenem as they produce carbapenamase known as KPC still low in this ICU.

Table 1: Different microorganisms isolated from the lower respiratory tract specimen from intensive care unit patients

\begin{tabular}{lccc}
\hline Organism & BAL & Sputum/Tracheal aspirate & Total (\%) \\
\hline A. baumannii & 4 & 32 & $36(24.2)$ \\
S. aureus & 4 & 27 & $31(20.7)$ \\
P. aeruginosa & 5 & 24 & $29(19.2)$ \\
K. pneumoniae & 3 & 26 & $29(19.2)$ \\
E. coli & 5 & 16 & $21(14)$ \\
Candida albicans & 4 & 16 & $20(13)$ \\
Coag-ve S. aureus & 2 & 16 & $18(11.5)$ \\
Burkholderia.cepacia & 2 & 13 & $15(10)$ \\
Enterococcus & 2 & 7 & $\mathbf{9 ( 6 . 1 )}$ \\
\hline Total & $31(14)$ & $177(82)$ & 208 \\
\hline
\end{tabular}

Table 2: Antimicrobial resistance rates (\%) for the micro organism recovered from lower respiratory tract secretions of intensive care unit patients.

\begin{tabular}{|c|c|c|c|c|c|c|}
\hline $\begin{array}{l}\text { Anti } \\
\text { microbials }\end{array}$ & $\begin{array}{c}\text { A. } \\
\text { baumannii (36) }\end{array}$ & $\begin{array}{c}P . \\
\text { aeruginosa (26) }\end{array}$ & $\begin{array}{c}\text { K. } \\
\text { pneumonia (26) }\end{array}$ & $\begin{array}{c}S . \\
\text { aureus (31) }\end{array}$ & $\begin{array}{c}E . \\
\text { coli }(21)\end{array}$ & $\begin{array}{c}\text { Coag-ve } \\
\text { Staph (18) }\end{array}$ \\
\hline Amikacin & 87.5 & 45 & 15 & ND & 10 & ND \\
\hline Gentamycin & 87 & 55 & 30 & 32 & 35 & 75 \\
\hline Tobramycin & 88 & 50 & ND & ND & ND & ND \\
\hline Amoxiclave & ND & ND & 65 & 90 & 90 & 85 \\
\hline \multicolumn{7}{|l|}{ Piperacillin/ } \\
\hline Taz & 86 & 30 & 16 & ND & ND & ND \\
\hline Cefepime & 93 & 31 & 36 & ND & 36 & ND \\
\hline Aztrenom & 93 & 39 & ND & ND & ND & ND \\
\hline Ceftazidime & 90 & 61 & ND & ND & ND & ND \\
\hline Meropenem & 89 & 40 & 13 & ND & ND & ND \\
\hline Imipenem & 91 & 40 & 16 & ND & ND & ND \\
\hline Ciprofloxacin & 90 & ND & 52 & 50 & 83 & 87 \\
\hline Cotrimoxazole & 90 & ND & 60 & 26 & 43 & 37.5 \\
\hline Tetracycline & 83 & ND & 55 & 50 & 56 & 40 \\
\hline Vancomycin & ND & ND & ND & 0 & 0 & 0 \\
\hline Linezolid & ND & ND & ND & 0 & 0 & 0 \\
\hline Colistin & 0 & 0 & 0 & ND & ND & ND \\
\hline
\end{tabular}




\section{Discussion:}

In our study, Acenetobactor baumanni (24.2\%) being the most common isolate followed by Staph. aereus (20.7\%) P. aeruginosa $(19.2 \%)$ and $K$. pneumoniae (19.2\%) Canndida (13.\%) Coagulase negative staph (11.5\%), Burkholderioa cepacia $(10 \%)$. A. baumanii is $(83-93 \%)$ is resistant to common antibiotics eg. meropenem, imipenem, ceftazidime, cefepime, ciprofloxacin, pipercilline with tazobactum, amikecin \& gentamicin \& it has no way to treat without colistin. As acinetobacter are generally resistant to most classes of antibiotics and emergence of carbapenem resistance strains are alarming in different parts of the world \& threat to the effective management of this infection. ${ }^{11}$

The third most pathogen in ICU $P$. aeruginosa $(30-66 \%)$ to different common drugs and it is $61 \%$ resistant to ceftazidime, 45 to $50 \%$ to aminoglycoside, $40 \%$ to carbapenem, $30 \%$ to pipercillin tazobactum, $31 \%$ to cefepime, $39 \%$ to aztreonam. ${ }^{10}$ So empirical treatment of suspected pseudomonas is better with pipercillin tazobactum or aminoglycoside.

K. pneumoniae (15-65\%) is resistant to common antibiotics $52 \%$ resistant to ciprofloxacin, 15 to $30 \%$ to aminoglycoside \& 13 to $16 \%$ to carbapenem which is still low in our study ICU and suggested to with judicious use of aminoglycosides \& carbapemems. We frequently use eftazidim, amikacin meropenem and piperacillin-tazobactum for pseudomonas infection. In our study we found $61 \%$ pseudomonas is resistant to ceftazidim, $45 \%$ to Amikacin, $55 \%$ to gentamycin and $40 \%$ to meropenem. The most sensitive antibiotic to pseudomonas is colistin followed by piperacillin -tazobactum and cefepime.

For MRSA, once a colony is established in an individual, it is almost impossible to eradicate, but can be suppressed for a short time which reduces the risk of the individual and hospital. ${ }^{9}$ Healthcare personnel should undergo screening and suppression therapy for MRSA routinely. ${ }^{9}$ MRSA \& E. Coli are most sensitive to vancomycine \& Linazolid and no resistance was found in our study. The second option to treat these organisms are aminoglycoside $\&$ co trimoxazole.

Candida species both albicans \& non albicans was the $5^{\text {th }}$ frequent isolated organism found in our study \& mostly immunocompromised patient with DM, poor nutritional status, use of high dose steroids \& broad spectrum antibiotics. ${ }^{12}$ they lack ideal treatment option. A vaccine is being on pipeline which would protect life threatening fungal infection. ${ }^{13}$

In 2006-2007 a study in ICU of BIRDEM showed major organisms were pseudomonas (29.1\%), Acinetobacter 27.5\%, candida (12.8\%), E coli (10.3\%), klebsialla (9.7\%). 75\% pseudomonas was resistance to $3^{\text {rd }}$ generation cephalosporin and $79.1 \%$ to quinolones \& 58.9\% to carbapenem. In 2005 same ICU reported $82 \%$ pseudomonas resistant to cephalosporins and $48 \%$ to Quinolone \& $36 \%$ to carbapenem. ${ }^{14}$ Similar observations were made by other investigators in different Asian countries that the most frequent pathogens in ICU were A. baumannii, P. aeruginosa,
K. pneumoniae, E. coli, Staph. aereus. Carbapenems are frequently used as a last choice in treating serious infections in ICU.

In our study, $90 \%$ isolates of Acinetobacter spp., $40 \%$ isolates of $P$. aeruginosa, and $15 \%$ isolates of Klebsiella spp. were resistant to carbapenem. These findings arises a question of KPC gene sread and suggests for judicious use of carbapenem in ICU. Another important observation of our study was that Tetracycline and Cotrimoxazole, the old drugs not used in empirical treatment in ICUs, shows moderate sensitivity to Klebsiella spp., Staph. aureus and E. coil.

In some US Hospitals pathogens like Acenetobactor Baumanni and Enterococcus faecalis are virtually untreatable because of acquisition of multiple determinant of resistance to antimicrobial agents. ${ }^{13}$ Vancomycin resistant Staph. Aureus (VRSA) is also becoming a big challenge to ICU management in in some countries. Further studies are required to evaluate the use of Carbapenem and Colistin for ICU patients because of least number of reserved antibiotics remaining. So, judicious use of older and newer antimicrobial agents is essential to prevent the emergence of multidrug resistant bacteria in the ICU.

\section{Conclusion:}

Highly resistant Acinetobacter infection can be controlled by strictly cleaning fomites, instruments and sometime temporary closure of the unit. Skin disinfection of both patients and staff with chlorohexidine or polymixin is necessary. Biofilm producing pseudomonas survive in fomites and foems causes microabscess in alveolar wall and relapses with necrosis and needs strict preventive measures. Coliforms have more than 500 classes of ESBL enzymes, inappropriate antibiotic use and frequent skin contamination of both patients and staff with feaces of the patient can spread the infection. Compulsory hand washing of staff and visitors and rigorous cleaning of environment can help in preventive measures. Plasmid Profile Analysis (PPA) can help in identifying the source of infection. ${ }^{15}$

\section{References:}

1. Vincent JL, et al. prevalence of nosocomial infection in Europe. JAMA 1995; 274-44.

2. Chestre $\mathrm{J}$, et al. Ventilator associated pneumonia. Am J Res Crit Care Med 2002; 165: 867-903.

3. Radji M, et at. Antibiotic sensitivity pattern of bacterial pathogen in the ICU of fatmawati hospital in Indonesia. Asian Pacific Journal of tropical biomedicine 2011; 39-42.

4. Bhaumik P, et al. Bacteriological profile and antibiogram of gram negative organism isolated from medical and neurology ICU with special reference to MDR organism. National Journal of medical Research India; 22494995.

5. Fridkin SK. Increasing prevalence of antimicrobial resistance in intensive care units. Critical Care Medicine 2001; 29: 64-68.

6. Shehabi AA, et al. Microbial infection \& antibiotic resistance pattern among Indian ICU pts. Eastern Mediterranean health journal $1996 ; 2: 515-520$ 
Bangladesh Crit Care J September 2014; 2 (2): 53-56

7. Tuller MS, et al. Bacterial profile and antimicrobial susceptibility pattern in catheter related nosocomial infections. Journal of post graduate medicine 1998; 44: 7-13.

8. Talbot GH, et al. Bad bugs needs an update on development pipeline from antimicrobial availability Task force. Infectious disease society of America. Clinical Infectious disease 2006, 42: 657-658.

9. Bromley L. Microbiology for anaesthetist. Antibiotic prophylaxis in Surgery. ATOTW 2011; 2-7.

10. Forbes BA et al. Baily \& Scott's diagnostic microbiology. $12^{\text {th }}$ edition. 2007.

11. Mulvey MR et al. Antimicrobial resistance in hospitals: How concerned should we be? Canadian Medical Association Journal 2009; 180: 408-415.
12. Barai $\mathrm{L}$, et al. Bacterial profile \& their anti microbial resistance pattern in an ICU of Tertiary Care Hospital in Dhaka. Ibrahim Medical Journal 2010; 6: 66-69.

13. Scott G. Antibiotic Resistance. Medicine 2009; 37(10):551-555.

14. Basunia MRA, et al. Microbial Pathogens \& antibiotic sensitivity at ICU of IRDEM. A retrospective study. Bangladesh Journal of Medicine 2005; 16: 14-20.

15. Mohiuddin $\mathrm{M}$, et al. Microbiology of Nosocomial Infection in Tertiary Care Hospital of Dhaka City and its impact. Bangladesh Journal of Med 2010; 4 (02): 32-38. 